\title{
KÄHLER STRUCTURES ON COMPACT SOLVMANIFOLDS
}

\author{
CHAL BENSON AND CAROLYN S. GORDON
}

(Communicated by Jonathan M. Rosenberg)

\begin{abstract}
In a previous paper, the authors proved that the only compact nilmanifolds $\Gamma \backslash G$ which admit Kähler structures are tori. Here we consider a more general class of homogeneous spaces $\Gamma \backslash G$, where $G$ is a completely solvable Lie group and $\Gamma$ is a cocompact discrete subgroup. Necessary conditions for the existence of a Kähler structure are given in terms of the structure of $G$ and a homogeneous representative $\omega$ of the Kähler class in $H^{2}(\Gamma \backslash G ; \mathbb{R})$. These conditions are not sufficient to imply the existence of a Kähler structure. On the other hand, we present examples of such solvmanifolds that have the same cohomology ring as a compact Kähler manifold. We do not know whether some of these solvmanifolds admit Kähler structures.
\end{abstract}

\section{INTRODUCTION}

In this paper we consider compact homogeneous spaces of the form $\Gamma \backslash G$, where $G$ is a connected and simply connected Lie group and $\Gamma$ is a discrete cocompact subgroup. When $G$ is a nilpotent Lie group, one calls $\Gamma \backslash G$ a nilmanifold, and when $G$ is solvable, $\Gamma \backslash G$ is a solvmanifold. (Many authors do not require nilmanifolds and solvmanifolds to be compact. Also note that there are compact homogeneous spaces of solvable Lie groups that are not quotients by discrete subgroups.) We will refer to a positive-definite Kähler metric as a Kähler structure. We remark that in contrast to Theorems 1 and 2 below, many solvmanifolds and nilmanifolds admit indefinite Kähler metrics. In a previous paper, we proved the following result.

Theorem 1. If a nilmanifold $\Gamma \backslash G$ admits a Kähler structure, then $G$ is an abelian group and $\Gamma \backslash G$ is a torus. (See [1, 2, 9], and [11].)

We will describe a generalization of Theorem 1 to a class of solvmanifolds. Let $\mathfrak{g}$ denote the Lie algebra of $G$ and $\mathfrak{n}$ the derived algebra, $\mathfrak{n}=[\mathfrak{g}, \mathfrak{g}]$ (that is, the linear space of all brackets $[X, Y])$. Recall that $\mathfrak{g}$ is solvable if and only if $\mathfrak{n}$ is nilpotent. One says that $\mathfrak{g}$ is completely solvable if $\operatorname{ad}(X): \mathfrak{g} \rightarrow \mathfrak{g}$ has

Received by the editors May 4, 1989.

1980 Mathematics Subject Classification (1985 Revision). Primary 53C55; Secondary 53C15, 22E25, 57T15.

The research of the first author was partially supported by a University of Missouri Weldon Springs Grant. The research of the second author was partially supported by NSF grant \#8502084 . 
only real eigenvalues for each $X \in \mathfrak{g}$. Equivalently, $\mathfrak{g}$ is isomorphic to a subalgebra of the (real) upper triangular matrices in $\operatorname{gl}(n, \mathbb{R})$ for some $n$. A given completely solvable Lie group $G$ may or may not have any cocompact discrete subgroups. For $G$ to admit a cocompact discrete subgroup, it is necessary that $G$ be unimodular.

Theorem 2. If $G$ is completely solvable and $\Gamma \backslash G$ is a solvmanifold that admits a Kähler structure, then

(i) there is an abelian complement $\mathfrak{a}$ in $\mathfrak{g}$ of the derived algebra $\mathfrak{n}=[\mathfrak{g}, \mathfrak{g}]$,

(ii) $\mathfrak{a}$ and $\mathfrak{n}$ are even dimensional;

(iii) the center of $\mathfrak{g}$ intersects $\mathfrak{n}$ trivially;

(iv) the Kähler form is cohomologous to a left invariant symplectic form $\omega=$ $\omega_{0}+\omega_{1}$, where $\mathfrak{n}=\operatorname{ker}\left(\omega_{0}\right)$ and $\mathfrak{a}=\operatorname{ker}\left(\omega_{1}\right)$ (i.e., $\mathfrak{a}$ and $\mathfrak{n}$ are symplectic and $\omega$-orthogonal subspaces of $(\mathfrak{g}, \omega))$;

(v) both $\omega_{0}$ and $\omega_{1}$ are closed but not exact in $\mathfrak{g}$ (and also in $\mathfrak{a}$ and $n$ );

(vi) the adjoint action of $\mathfrak{a}$ on $\mathfrak{n}$ is by infinitesimal symplectic automorphisms of $(\mathfrak{n}, \omega)$.

Thus $G$ is a semidirect product $G=A \ltimes N$, where $A$ is a connected abelian subgroup and $N$ is the (nilpotent) commutator subgroup. Moreover, $N$ admits a left-invariant symplectic structure, and the action of $A$ on $N$ is by symplectomorphisms.

Theorem 2 generalizes Theorem 1 since (iii) fails when $\mathfrak{g}$ is nilpotent but nonabelian.

It is important to note that the Kähler structures in Theorems 1 and 2 need not arise from left- $G$-invariant Kähler structures on $G$. The general homogeneous Kähler manifold is now well understood after a lengthy research program culminating in recent work of Dorfmeister and Nakajima [3]. Hano showed that if a unimodular Lie group $G$ admits a left-invariant Kähler structure, then $G$ is solvable [8]. Moreover, as in Theorem 2, $G$ splits as a semidirect product of an abelian subgroup with the commutator subgroup $N=[G, G]$ and in this case $N$ is also abelian. However, $G$ will not be completely solvable unless it is abelian. Indeed, with notation as in the statement of Theorem 2, Hano shows that $\operatorname{ad}(X)(Y)=\nabla_{X}(Y)$ for $x \in \mathfrak{a}$ and $Y \in \mathfrak{n}$. Here $\nabla$ denotes the Riemannian connection on $G$. Compatibility of $\nabla$ with the Riemannian metric implies that $\operatorname{ad}(X)$ is skew-symmetric with respect to the inner product obtained by restricting this metric to $\mathfrak{n}$. It follows that $\operatorname{ad}(X): \mathfrak{n} \rightarrow \mathfrak{n}$ has only pure imaginary eigenvalues. One calls such a group solvable of type $R$. In particular, if $G$ is a completely solvable, unimodular, and homogeneous Kähler structure, then $G$ is nilpotent and in fact abelian. We remark that a nonunimodular completely solvable Lie group may admit a homogeneous Kähler structure. For examples we refer the reader to [5]. Such examples played an important role in the subsequent general classification program.

In view of the above discussion, it seems reasonable to conjecture that the only Kählerian solvmanifolds $\Gamma \backslash G$ with $G$ completely solvable are tori. This 
may be true, but we have only been able to prove the much weaker necessary conditions in Theorem 2. In $\S 3$, we present some examples showing where the difficulties lie.

The proof of Theorem 1 given in [1] involved showing that the Hard Lefschetz Theorem fails for any symplectic structure on a nontoral nilmanifold $\Gamma \backslash G$. In [11], McDuff shows that this failure of the Hard Lefschetz Theorem is a consequence of the existence of a certain circle action on $\Gamma \backslash G$. Hasegawa proved Theorem 1 independently by showing that a minimal model of the deRham complex for $\Gamma \backslash G$ could not be formal [9]. Cordero, Fernandez, and Gray have shown that every nonabelian nilmanifold admits nontrivial Massey triple products [2]. We recently learned that Koszul was aware of Theorem 1 (see the note in [8]), although he never published it. The authors are grateful to Dusa McDuff for pointing this out to them.

All these proofs use Nomizu's Theorem, which asserts that the Lie algebra cohomology $H^{*}(\mathfrak{g})$ of the complex $\Lambda\left(\mathfrak{g}^{*}\right)$ of left-invariant forms on a nilpotent Lie group $G$ is isomorphic to the deRham cohomology $H^{*}(\Gamma \backslash G ; \mathbb{R})$ of an associated nilmanifold [12]. Nomizu's Theorem was generalized to completely solvable Lie groups $G$ by Hattori [10]. This result will be used in the next section.

\section{Proof of Theorem 2}

Suppose that $\Gamma \backslash G$ has a Kähler structure and let $n=2 m=\operatorname{dim}(\mathfrak{g})$. As in the proof of Theorem 1 given in [1], it suffices to show that for any left-invariant symplectic form $\omega$, the map

$$
\wedge[\omega]^{m-1}: H^{1}(\mathfrak{g}) \rightarrow H^{n-1}(\mathfrak{g})
$$

is an isomorphism only if conditions (i) through (vi) hold. Indeed, Hattori's generalization of Nomizu's Theorem shows that the cohomology class of the Kähler form has a left-invariant representative. This will be a symplectic structure satisfying the Hard Lefschetz Theorem (see [6]).

We view the dual space $\mathfrak{g}^{*}$ of $\mathfrak{g}$ as the space of left-invariant 1-forms. Any choice of vector space complement $\mathfrak{a}$ of $\mathfrak{n}$ in $\mathfrak{g}$ (we will make a particular choice later) allows us to decompose $\mathfrak{g}^{*}$ as a vector space sum $\mathfrak{g}^{*}=\mathfrak{a}^{*}+\mathfrak{n}^{*}$, where $\mathfrak{a}^{*}=\left\{\eta \in \mathfrak{g}^{*} \mid \eta(\mathfrak{n})=0\right\}$ and $\mathfrak{n}^{*}=\left\{\eta \in \mathfrak{g}^{*} \mid \eta(\mathfrak{a})=0\right\}$. Note that $\mathfrak{a}^{*}$ is independent of the choice of $\mathfrak{a}$. Let $\ell=\operatorname{dim}(\mathfrak{n})$ and $k=\operatorname{dim}(\mathfrak{a})=n-\ell$. Denote $\Lambda^{i}\left(\mathfrak{a}^{*}\right) \wedge \Lambda^{j}\left(\mathfrak{n}^{*}\right)$ by $\Lambda^{i, j}$ so that

$$
\Lambda^{p}\left(\mathfrak{g}^{*}\right)=\sum_{i+j=p} \Lambda^{i, j}
$$

Recall that for $\alpha \in \mathfrak{g}^{*}$ and $X, Y \in \mathfrak{g}$, we have $d \alpha(X, Y)=-\alpha([X, Y])$. Since $\mathfrak{n}=[\mathfrak{g}, \mathfrak{g}]$, we find that $d\left(\Lambda^{1,0}\right)=0$ and that $d: \Lambda^{0,1} \rightarrow \Lambda^{2}\left(\mathfrak{g}^{*}\right)$ is one-to-one. Hence,

$$
H^{1}(\Gamma \backslash G) \cong H^{1}(\mathfrak{g})=\Lambda^{1,0}
$$


To compute $H^{n-1}(\mathfrak{g})$, note that any $(n-1)$-form $\lambda$ is closed. Indeed, we can write $\lambda=i(X) \Omega$ for some $X \in \mathfrak{g}$ and $\Omega \in \Lambda^{n}\left(\mathfrak{g}^{*}\right)$. Here $i(X)$ denotes the interior product. An easy computation shows that $d \lambda=-\operatorname{tr}(\operatorname{ad}(X)) \Omega$. Since unimodularity of $\mathfrak{g}$ means that $\operatorname{tr}(\operatorname{ad}(X))=0$ for all $X \in \mathfrak{g}$, we see that $\lambda$ is closed. Next we claim that $d\left(\Lambda^{n-2}\left(\mathfrak{g}^{*}\right)\right) \subseteq \Lambda^{k, \ell-1}$. We have

$$
\Lambda^{n-2}\left(\mathfrak{g}^{*}\right)=\Lambda^{k, \ell-2}+\Lambda^{k-1, \ell-1}+\Lambda^{k-2, \ell} .
$$

As noted above, $d\left(\Lambda^{1,0}\right)=0$, so if $\lambda \in \Lambda^{i, 0}$ and $\mu \in \Lambda^{0, j}$, then $d(\lambda \wedge \mu)=$ $\pm \lambda \wedge d \mu$. Hence,

$$
d: \Lambda^{i, j} \rightarrow \Lambda^{i+2, j-1}+\Lambda^{i+1, j}+\Lambda^{i, j+1}
$$

In particular,

$$
d: \Lambda^{k, \ell-2} \rightarrow \Lambda^{k, \ell-1}
$$

Since $\mathfrak{n}$ is unimodular, the argument above shows that $d_{\mathfrak{n}}\left(\Lambda^{\ell-1}\left(\mathfrak{n}^{*}\right)\right)=0$, where $d_{\mathfrak{n}}$ is the exterior derivative on $\Lambda\left(\mathfrak{n}^{*}\right)$. That is, for $\mu \in \Lambda^{k-1, \ell-1}$, the $\Lambda^{k-1, \ell}$-component of $d \mu$ is zero and thus

$$
d: \Lambda^{k-1, \ell-1} \rightarrow \Lambda^{k, \ell-1} .
$$

For $\eta \in \Lambda^{0, \ell}$ write $d \eta=(d \eta)_{1, \ell}+(d \eta)_{2, \ell-1}$ where $(d \eta)_{i, j} \in \Lambda^{i, j}$. For $A \in \mathfrak{a}$ and $\left\{X_{1}, \ldots, X_{\ell}\right\}$ a basis of $\mathfrak{n}$, we have

$$
\begin{aligned}
d \eta\left(A, X_{1}, \ldots, X_{\ell}\right) & =-\operatorname{tr}\left(\left.\operatorname{ad}(A)\right|_{\mathfrak{n}}\right) \eta\left(X_{1}, \ldots, X_{\ell}\right) \\
& =-\operatorname{tr}(\operatorname{ad}(A)) \eta\left(X_{1}, \ldots, X_{\ell}\right) .
\end{aligned}
$$

(The second equality follows from $[\mathfrak{g}, \mathfrak{g}]=\mathfrak{n}$.) Since $\mathfrak{g}$ is unimodular, $(d \eta)_{1, \ell}=0$ and $d: \Lambda^{0, \ell} \rightarrow \Lambda^{2, \ell-1}$. Thus

$$
d: \Lambda^{k-2, \ell} \rightarrow \Lambda^{k, \ell-1} .
$$

Together, Equations (2.2), (2.4), (2.5), and (2.6) show that $d\left(\Lambda^{n-1}\left(\mathfrak{g}^{*}\right)\right) \subseteq$ $\Lambda^{k, \ell-1}$ as claimed. Since $d\left(\Lambda^{n-1}\left(\mathfrak{g}^{*}\right)\right)=0$, we now have

$$
\operatorname{dim}\left(H^{n-1}(\mathfrak{g})\right) \geq \operatorname{dim}\left(\Lambda^{n-1}\left(\mathfrak{g}^{*}\right)\right)-\operatorname{dim}\left(\Lambda^{k, \ell-1}\right)=n-\ell=k .
$$

On the other hand, Poincaré duality for $\Gamma \backslash G$ shows

$$
\operatorname{dim}\left(H^{n-1}(\mathfrak{g})\right)=\operatorname{dim}\left(H^{1}(\mathfrak{g})\right)=\operatorname{dim}\left(\Lambda^{1,0}\right)=k .
$$

It follows that

$$
\text { every element of } \Lambda^{k, \ell-1} \text { is exact and } H^{n-1}(\mathfrak{g}) \cong \Lambda^{k-1, \ell} \text {. }
$$

Now let $\omega \in \Lambda^{2}\left(\mathfrak{g}^{*}\right)$ be a left-invariant symplectic form for which $\Lambda[\omega]^{m-1}$ : $H^{1}(\mathfrak{g}) \rightarrow H^{n-1}(\mathfrak{g})$ is an isomorphism. For any $X \in \mathfrak{n}$,

$$
(i(X) \omega) \wedge \omega^{m-1}=(1 / m) i(X) \omega^{m} \in \Lambda^{k, \ell-1}
$$


and hence $(i(X) \omega) \wedge \omega^{m-1}$ is exact by (2.7). Our hypothesis on $\omega$ together with Equation 2.1 imply that $i(X) \omega \notin \Lambda^{1,0}$. This means there must exist some $Y \in \mathfrak{n}$ for which $\omega(X, Y) \neq 0$ and hence $\left.\omega\right|_{\mathfrak{n} \times \mathfrak{n}}$ is nondegenerate.

We now choose $\mathfrak{a}$ to be the $\omega$-orthogonal complement to $\mathfrak{n}$,

$$
\mathfrak{a}=\{A \in \mathfrak{g} \mid \omega(A, \mathfrak{n})=0\} .
$$

One has $\omega \in \Lambda^{2,0}+\Lambda^{0,2}$, say $\omega=\omega_{2,0}+\omega_{0,2}$ where $\omega_{2,0}$ and $\omega_{0,2}$ are symplectic forms on the vector spaces $\mathfrak{a}$ and $\mathfrak{n}$. Since $\omega$ and all elements of $\Lambda^{2,0}$ are closed, $\omega_{2,0}$ and $\omega_{0,2}$ are both closed. Neither is exact since $[\omega]^{m} \neq 0$. Setting $\omega_{0}=\omega_{2,0}$ and $\omega_{1}=\omega_{0,2}$ we obtain (ii), (iv), and (v).

We next show $\mathfrak{a}$ is abelian. Let $A, B \in \mathfrak{a}$ and $X \in \mathfrak{n}$. Then

$$
0=d \omega(A, B, X)=-\omega([A, B], X)+\omega([A, X], B)-\omega([B, X], A) .
$$

The last two terms on the right are zero since $\left.\omega\right|_{\mathfrak{a} \times \mathfrak{n}}=0$. Hence $\omega([A, B], X)=$ 0 for all $X \in \mathfrak{n}$. By nondegeneracy of $\omega$ on $\mathfrak{n} \times \mathfrak{n}$, we find that $[A, B]=0$ so that $\mathfrak{a}$ is abelian.

To prove (vi), we observe that for $A \in \mathfrak{a}$ and $X, Y \in \mathfrak{n}, \omega(A,[X, Y])=0$ so

$$
0=d \omega(A, X, Y)=-\omega([A, X], Y)-\omega(X,[A, Y]) .
$$

Thus $\left.\operatorname{ad}(A)\right|_{\mathfrak{n}}$ belongs to the symplectic Lie algebra $\left(\mathfrak{n}, \omega_{1}\right)$.

Suppose $Z \in \mathfrak{n} \cap \mathfrak{Z}(\mathfrak{g}), Z \neq 0$. Then $\eta=i(Z) \omega$ is closed and we obtain $[\eta] \neq 0$ in $H^{1}(\mathfrak{g})$. However $\omega^{m-1} \wedge \eta=(1 / m) i(Z) \omega^{m} \in \Lambda^{k, \ell-1}$ is exact (see (2.7)) contradicting the assumption that $\wedge[\omega]^{m-1}: H^{1}(\mathfrak{g}) \rightarrow H^{n-1}(\mathfrak{g})$ is an isomorphism. This proves condition (iii).

The final paragraph in the statement of Theorem 2 follows from (i) to (vi) since $G$ is simply connected.

Remarks.

(i) We assumed that $G$ was completely solvable so that we could use Hattori's Theorem. The conclusions of Theorem 2 remain true if $\Gamma \backslash G$ is any compact homogeneous space (with $\Gamma \leq G$ discrete) for which there is an algebra isomorphism $H^{*}(\Gamma \backslash G) \cong H^{*}(\mathfrak{g})$. Indeed, existence of a cocompact discrete subgroup $\Gamma$ implies unimodularity of both $\mathfrak{g}$ and $\mathfrak{n}=[\mathfrak{g}, \mathfrak{g}]$, which is all we used in the proof. Of course $\mathfrak{n}$ will not be nilpotent unless $\mathfrak{g}$ is solvable. See [13] for some other solvable contexts where $H^{*}(\Gamma \backslash G) \cong H^{*}(\mathfrak{g})$.

(ii) In [11], McDuff calls a compact symplectic manifold $\left(M^{2 m}, \omega\right)$ a Lefschetz manifold when $\wedge[\omega]^{m-1}: H^{1}(M) \rightarrow H^{2 m-1}(M)$ is an isomorphism. The proof of Theorem 2 shows that (i) to (vi) are necessary conditions for a given symplectic solvmanifold $\Gamma \backslash G$, with $G$ completely solvable, to be a Lefschetz manifold (replacing the words "Kähler form" by "symplectic form" in (iv)). The same reasoning also shows that conditions (i) to (vi) are sufficient for $\Gamma \backslash G$ to be a Lefschetz manifold. 
(iii) The subalgebra $\mathfrak{a}$ in Theorem 2 is uniquely determined by the choice of left-invariant representative $\omega$ of the Kähler class. The authors have shown that different choices of $\omega$ will yield subalgebras $\mathfrak{a}$ that differ by the ad-action of $G$ on $\mathfrak{g}$. On the group level, this means that the abelian subgroup $A \leq G$ is determined up to inner automorphism by the Kähler structure.

\section{EXAMPLES}

In this section we present certain completely solvable Lie algebras $\mathfrak{g}$ that satisfy the conditions of Theorem 2 for some symplectic structure $\omega \in \Lambda^{2}\left(\mathfrak{g}^{*}\right)$. As remarked at the end of $\S 2$, the Lefschetz map from $H^{1}(\mathfrak{g})$ will be an isomorphism in each case. However, one can consider additional conditions on the cohomology of a compact Kähler manifold. In particular, one can examine the Hodge relations, look for vanishing of Massey triple products, and compute the Lefschetz map in higher degrees.

In Example 1, we explicitly construct a cocompact discrete subgroup $\Gamma$ of the Lie group $G$ corresponding to $\mathfrak{g}$. In Examples 2 and 3, we have been unable to determine whether $G$ admits a cocompact subgroup. We note, however, that in both cases $\mathfrak{g}$ is "strongly unimodular"; the restriction of $\operatorname{ad}(X)$ to $\mathfrak{m}^{(i)}$ has trace zero for each term $\mathfrak{m}^{(i)}$ in the descending central series for the nilradical $\mathfrak{m}$ of $\mathfrak{g}$. This is a necessary condition for the existence of a cocompact discrete subgroup of $G$.

Example 1. Let $\mathfrak{g}$ be the Lie algebra direct sum $\mathbb{R} \oplus \mathfrak{g}_{0}$ where $\mathfrak{g}_{0}$ has basis $\{H, X, Y\}$ with nontrivial structure equations

$$
\begin{aligned}
& {[H, X]=X} \\
& {[H, Y]=-Y .}
\end{aligned}
$$

Letting $Z$ span the ideal $\mathbb{R}$, we have $\mathfrak{a}=\operatorname{Span}(Z, H)$ and $\mathfrak{n}=\operatorname{Span}(X, Y)$. Both $\mathfrak{a}$ and $\mathfrak{n}$ are abelian and $\mathfrak{Z}(\mathfrak{g})=\operatorname{Span}(Z) \subset \mathfrak{a}$. The simply connected Lie group $G_{0}$ corresponding to $\mathfrak{g}_{0}$ can be written as $\mathbb{R} \ltimes \mathbb{R}^{2}$, where $\mathbb{R}$ acts on $\mathbb{R}^{2}$ via

$$
t \mapsto A_{t}=\left(\begin{array}{cc}
e^{t} & 0 \\
0 & e^{-t}
\end{array}\right) .
$$

The simply connected Lie group $G$ corresponding to $\mathfrak{g}$ is given by $G=\mathbb{R} \times G_{0}$.

We now construct a cocompact discrete subgroup $\Gamma_{0}$ of $G_{0}$. We will then take $\Gamma=\Gamma_{0} \times \mathbb{Z}$, a cocompact discrete subgroup of $G$. To construct $\Gamma_{0}$, it suffices to find some $t_{0} \neq 0$ with $A_{t_{0}}$ conjugate to an element of $\operatorname{SL}(2, \mathbb{Z})$. Indeed, we could then find a lattice $L$ in $\mathbb{R}^{2}$ which is invariant under $A_{t_{0}}^{n}$ for all $n \in \mathbb{Z}$ and take $\Gamma_{0}=\left(t_{0} \mathbb{Z}\right) \ltimes L$. To obtain $t_{0}$, choose any element $B$ of $\operatorname{SL}(2, \mathbb{Z})$ with distinct real eigenvalues, say $\lambda$ and $1 / \lambda$, and take $t_{0}=\log (\lambda)$.

Let $\{\lambda, \gamma, \mu, \nu\}$ denote the basis for $\mathfrak{g}^{*}$ dual to $\{Z, H, X, Y\}$. Then

$$
d \mu=-\gamma \wedge \mu
$$


and

$$
d \nu=\gamma \wedge \nu
$$

The form

$$
\omega=\lambda \wedge \gamma+\mu \wedge \nu
$$

is a symplectic structure satisfying the conditions of Theorem 2. In particular, $\wedge[\omega]: H^{1}(\mathfrak{g}) \rightarrow H^{3}(\mathfrak{g})$ is an isomorphism.

We have $\pi_{1}\left(\Gamma_{0} \backslash G_{0}\right)=\Gamma_{0}$ and hence $H^{1}\left(\Gamma_{0} \backslash G_{0} ; \mathbb{Z}\right) \cong \Gamma_{0} /\left[\Gamma_{0}, \Gamma_{0}\right] \cong \mathbb{Z}$. By Poincaré duality, we also have $H^{2}\left(\Gamma_{0} \backslash G_{0} ; \mathbb{Z}\right) \cong \mathbb{Z}$. Choose maps

$$
h_{1}: \Gamma_{0} \backslash G_{0} \rightarrow S^{1} \text { and } h_{2}: \Gamma_{0} \backslash G_{0} \rightarrow S^{2}
$$

representing the generators in $H^{1}\left(\Gamma_{0} \backslash G_{0} ; \mathbb{Z}\right)$ and $H^{2}\left(\Gamma_{0} \backslash G_{0} ; \mathbb{Z}\right)$. (The generator of $H^{2}\left(\Gamma_{0} \backslash G_{0} ; \mathbb{Z}\right)$ is represented by a map into $K(\mathbb{Z}, 2)=\mathbb{C P}^{\infty}$, which is homotopic to one whose image lies in the 2 skeleton $S^{2}$ since $\Gamma_{0} \backslash G_{0}$ is a 3-manifold and $\mathbb{C P}^{\infty}$ has no 3-cells.) The map

$$
f_{0}: \Gamma_{0} \backslash G_{0} \rightarrow S^{1} \times S^{2}, x \mapsto\left(h_{1}(x), h_{2}(x)\right)
$$

is a cohomology isomorphism and so is $f=i d \times f_{0}: \Gamma \backslash G \rightarrow T^{2} \times S^{2}$. Note that in this construction the Kähler class in $H^{2}\left(T^{2} \times S^{2} ; \mathbb{R}\right)$ pulls back to [ $\omega$ ] under $f$.

Since $T^{2} \times S^{2}$ is a Kähler manifold, we see that $\Gamma \backslash G$ is cohomologically Kähler. However, it is shown in [4] that $\Gamma \backslash G$ does not admit a complex structure (and hence admits no Kähler structure). This is done by appealing to classification theorems of Kodaira and Yau that are specific to complex dimension 2. Related examples in higher dimensions, such as $\Gamma_{0} \backslash G_{0} \times \Gamma_{0} \backslash G_{0}$ and $T^{3} \times \Gamma_{0} \backslash G_{0}$ in dimension 6 are also cohomologically Kähler, but we do not know whether any of these admit Kähler structures.

Example 2. Let $\mathfrak{g}=\operatorname{Span}\left(A, B, X_{1}, X_{2}, X_{3}, Z_{1}, Z_{2}, Z_{3}\right)$, where

$$
\begin{aligned}
{\left[X_{2}, X_{3}\right] } & =2 Z_{1}, \quad\left[X_{1}, X_{3}\right]=Z_{2}, \quad\left[X_{1}, X_{2}\right]=-Z_{3}, \\
{\left[A, X_{1}\right] } & =-X_{1}, \quad\left[A, X_{2}\right]=-2 X_{2}, \quad\left[A, X_{3}\right]=3 X_{3} \text { and } \\
{\left[A, Z_{1}\right] } & =Z_{1}, \quad\left[A, Z_{2}\right]=2 Z_{2}, \quad\left[A, Z_{3}\right]=-3 Z_{3} .
\end{aligned}
$$

Here $\mathfrak{g}=\mathfrak{a} \ltimes \mathfrak{n}$ where $\mathfrak{a}=\operatorname{Span}(A, B)$ and $\mathfrak{n}=\operatorname{Span}\left(X_{1}, X_{2}, X_{3}, Z_{1}, Z_{2}, Z_{3}\right)$. In this example, $\mathfrak{n}$ is two-step nilpotent and $\mathfrak{Z}(\mathfrak{g})=\operatorname{Span}(B) \subset \mathfrak{a}$. Denoting the dual basis for $\mathfrak{g}^{*}$ by $\left\{\alpha, \beta, \mu_{1}, \mu_{2}, \mu_{3}, \zeta_{1}, \zeta_{2}, \zeta_{3}\right\}$, one has

$$
d \mu_{1}=\alpha \wedge \mu_{1}, \quad d \mu_{2}=2 \alpha \wedge \mu_{2}, \quad d \mu_{3}=-3 \alpha \wedge \mu_{3}
$$

and

$$
\begin{gathered}
d \zeta_{1}=-\alpha \wedge \zeta_{1}-2 \mu_{2} \wedge \mu_{3}, \quad d \zeta_{2}=-2 \alpha \wedge \zeta_{2}-\mu_{1} \wedge \mu_{3} \\
d \zeta_{3}=3 \alpha \wedge \zeta_{3}+\mu_{1} \wedge \mu_{2}
\end{gathered}
$$


A symplectic structure satisfying the conditions of Theorem 2 is given by

$$
\omega=\alpha \wedge \beta+\left(\mu_{1} \wedge \zeta_{1}+\mu_{2} \wedge \zeta_{2}+\mu_{3} \wedge \zeta_{3}\right)=\omega_{0}+\omega_{1}
$$

One can compute that

$H^{1}(\mathfrak{g})=\operatorname{Span}([\alpha],[\beta])$,

$H^{2}(\mathfrak{g})=\operatorname{Span}\left(\left[\omega_{0}\right],\left[\omega_{1}\right]\right)$,

$H^{3}(\mathfrak{g})=\operatorname{Span}\left(\left[\alpha \wedge \omega_{1}\right],\left[\beta \wedge \omega_{1}\right]\right)$,

$H^{4}(\mathfrak{g})=\operatorname{Span}\left(\left[\omega_{0} \wedge \omega_{1}\right],\left[\omega_{1} \wedge \omega_{1}\right]\right)$,

$H^{5}(\mathfrak{g})=\operatorname{Span}\left(\left[\alpha \wedge \omega_{1}^{2}\right],\left[\beta \wedge \omega_{1}^{2}\right]\right)$,

$H^{6}(\mathfrak{g})=\operatorname{Span}\left(\left[\omega_{1}^{3}\right],\left[\omega_{0} \wedge \omega_{1}^{2}\right]\right)$

$H^{7}(\mathfrak{g})=\operatorname{Span}\left(\left[\alpha \wedge \mu_{1} \wedge \mu_{2} \wedge \mu_{3} \wedge \zeta_{1} \wedge \zeta_{2} \wedge \zeta_{3}\right],\left[\beta \wedge \mu_{1} \wedge \mu_{2} \wedge \mu_{3} \wedge \zeta_{1} \wedge \zeta_{2} \wedge \zeta_{3}\right]\right)$

$H^{8}(\mathfrak{g})=\operatorname{Span}\left(\left[\alpha \wedge \beta \wedge \mu_{1} \wedge \mu_{2} \wedge \mu_{3} \wedge \zeta_{1} \wedge \zeta_{2} \wedge \zeta_{3}\right]\right)$

The Lefschetz maps $\wedge\left[\omega^{3}\right]: H^{1}(\mathfrak{g}) \rightarrow H^{7}(\mathfrak{g}), \wedge\left[\omega^{2}\right]: H^{2}(\mathfrak{g}) \rightarrow H^{6}(\mathfrak{g})$ and $\wedge[\omega]: H^{3}(\mathfrak{g}) \rightarrow H^{5}(\mathfrak{g})$ are all isomorphisms. In fact, $H^{*}(\mathfrak{g})$ is the cohomology ring of a Kähler manifold. One way to see this is as follows.

A minimal model for the Kähler manifold $T^{2} \times \mathbb{C P}^{3}$ is given by the differential graded algebra (DGA)

$$
\mathfrak{M}=\Lambda(a, b, c, k)
$$

freely generated by $a, b, c$, and $k$, where $\operatorname{deg}(a)=1=\operatorname{deg}(b), \operatorname{deg}(c)=$ $2, \operatorname{deg}(k)=7$, and $d a=d b=d c=0, d k=c^{4}$ [7]. The Kähler form on $T^{2} \times \mathbb{C P}^{3}$ is represented by $a \wedge b+c$. However, $\mathfrak{M}$ is also the (nilpotent) minimal model for the $D G A \Lambda\left(\mathfrak{g}^{*}\right)$. Indeed, the $D G A$ map $\varphi: \mathfrak{M} \rightarrow \Lambda\left(\mathfrak{g}^{*}\right)$ given by $\varphi(a)=\alpha, \varphi(b)=\beta, \varphi(c)=\omega_{1}$ and $\varphi(k)=0$ induces an isomorphism on cohomology and sends $a \wedge b+c$ to $\omega$. This shows that there can be no cohomological obstructions to a manifold of the form $\Gamma \backslash G$ being Kähler, where $G$ is the simply connected Lie group with Lie algebra $\mathfrak{g}$.

Example 3. Consider $\mathfrak{g}=\operatorname{Span}\left(A, B, X_{1}, Y_{1}, Z_{1}, X_{2}, Y_{2}, Z_{2}\right)$, where

$$
\begin{aligned}
{\left[X_{1}, Y_{1}\right] } & =Z_{1}, \quad\left[X_{2}, Y_{2}\right]=Z_{2}, \\
{\left[A, X_{1}\right] } & =X_{1}, \quad\left[A, X_{2}\right]=-X_{2}, \\
{\left[A, Y_{1}\right] } & =-2 Y_{1}, \quad\left[A, Y_{2}\right]=2 Y_{2} \\
{\left[A, Z_{1}\right] } & =-Z_{1}, \quad\left[A, Z_{2}\right]=Z_{2} .
\end{aligned}
$$

Here $\mathfrak{n}=\operatorname{Span}\left(X_{1}, Y_{1}, Z_{1}, X_{2}, Y_{2}, Z_{2}\right), \mathfrak{a}=\operatorname{Span}(A, B)$ and $\mathfrak{Z}(\mathfrak{g})=\operatorname{Span}(B)$. In this example $\mathfrak{n}$ is two-step nilpotent. Denoting the dual basis for $\mathfrak{g}^{*}$ by 
$\left\{\alpha, \beta, \mu_{1}, \nu_{1}, \zeta_{1}, \mu_{2}, \nu_{2}, \zeta_{2}\right\}$, one has

$$
\begin{aligned}
d \alpha & =0=d \beta, \\
d \mu_{1} & =-\alpha \wedge \mu_{1}, \quad d \mu_{2}=\alpha \wedge \mu_{2}, \\
d \nu_{1} & =2 \alpha \wedge \nu_{1}, \quad d \nu_{2}=2 \alpha \wedge \nu_{2} \\
d \zeta_{1} & =a \wedge \zeta_{1}-\mu_{1} \wedge \nu_{1}, \quad d \zeta_{2}=-\alpha \wedge \zeta_{2}-\mu_{2} \wedge \nu_{2} .
\end{aligned}
$$

In this case we will show that no solvmanifold $\Gamma \backslash G$ associated with $\mathfrak{g}$ admits a Kähler structure. Suppose that $\Gamma \backslash G$ does in fact have a Kähler structure. The Kähler form would then be cohomologous to a left-invariant 2-form $\omega=$ $\omega_{0}+\omega_{1}$ as in Theorem 2. The collection $\left\{\mu_{1} \wedge \zeta_{1}, \mu_{2} \wedge \zeta_{2}, \mu_{1} \wedge \mu_{2}, \nu_{1} \wedge \nu_{2}\right\}$ is a basis for the 2 -forms on $\mathfrak{n}$ that are closed in $\mathfrak{g}$. Hence we must have

$$
\omega=a \alpha \wedge \beta+\left(b \mu_{1} \wedge \zeta_{1}+c \mu_{2} \wedge \zeta_{2}+e \mu_{1} \wedge \mu_{2}+f \nu_{1} \wedge \nu_{2}\right)
$$

for some constants $a, b, c, e, f$. Nondegeneracy of $\omega$ implies that $a \neq 0$, $b \neq 0, c \neq 0$, and $f \neq 0$. For any such $\omega$, the map $\wedge\left[\omega^{3}\right]: H^{1}(\mathfrak{g}) \rightarrow H^{7}(\mathfrak{g})$ is an isomorphism. However, one can show that $\wedge\left[\omega^{2}\right]: H^{2}(\mathfrak{g}) \rightarrow H^{6}(\mathfrak{g})$ is not an isomorphism for any such $\omega$. Indeed, one computes that for the closed nonexact form $\mu_{1} \wedge \mu_{2}$,

$$
\begin{aligned}
\left(\mu_{1} \wedge \mu_{2}\right) \wedge \omega^{2} & =2 a f \alpha \wedge \beta \wedge \mu_{1} \wedge \mu_{2} \wedge \nu_{1} \wedge \nu_{2} \\
& =d\left(2 a f \alpha \wedge \beta \wedge \mu_{1} \wedge \nu_{1} \wedge \zeta_{2}\right) .
\end{aligned}
$$

That is, the Hard Lefschetz Theorem cannot work simultaneously on both $H^{1}(\mathfrak{g})$ and $H^{2}(\mathfrak{g})$ for any symplectic structure $\omega$.

\section{ACKNOWLEDGMENT}

We thank David Webb for many helpful discussions concerning this work.

\section{REFERENCES}

1. C. Benson and C. Gordon, Kähler and symplectic structures on nilmanifolds, Topology 27 (1988), 513-518

2. L. A. Cordero, M. Fernández and A. Gray, Compact symplectic manifolds not admitting positive definite Kähler metrics, preprint.

3. J. Dorfmeister and K. Nakajima, The fundamental conjecture for homogeneous Kähler manifolds, Acta Math. 161 (1988), 23-70.

4. M. Fernández and A. Gray, Compact symplectic solvmanifolds not admitting complex structures, (to appear in J. Geom. Phys.).

5. S. G. Gindikin, I. I. Pjatecckii-Shapiro and E. B. Vinberg, Homogeneous Kähler manifolds, translation by A. Koranyi, in Geometry of homogeneous domains, Edizioni Cremonese, Rome, 1968.

6. P. Griffiths and J. Harris, Principles of algebraic geometry, John Wiley and Sons, New York, 1978.

7. P. Griffiths and J. Morgan, Rational homotopy theory and differential forms, Birkhäuser, Boston, 1981.

8. J. Hano, On Kählerian homogeneous spaces of unimodular Lie groups, Amer. J. Math., 79 (1957), 885-900. 
9. K. Hasegawa, Minimal models of nilmanifolds, preprint.

10. A. Hattori, Spectral sequence in the deRham cohomology of fibre bundles, J. Fac. Sci. Univ. Tokyo, Sect. 1, 8 (1960), 289-331.

11. D. McDuff, The momentum map for circle actions on symplectic manifolds, (to appear in $\mathrm{J}$. Geom. Phys.).

12. K. Nomizu, On the cohomology of homogeneous spaces of nilpotent Lie groups, Ann. Math. 59 (1954), 531-538.

13. M. S. Raghunathan, Discrete subgroups of Lie groups, Springer, Berlin and New York, 1972.

Department of Mathematics and Computer Science, University of Missouri, St. Louis, MisSOURI, 63121

Department of Mathematics, Washington University in St. Louis, St. Louis, Missouri, 63130 